\title{
Simulation of the Dynamic Behavior of Electric Power Steering Systems Using Dedicated Finite Elements*
}

\author{
François BESSON**, Guy FERRARIS***, Michèle GUINGAND*** \\ and Jean-Pierre de VAUJANY $* * *$ \\ ** JTEKT Europe, ZI du Broteau, BP1, F-69540 Irigny, France \\ E-mail: francois.besson@jtekt-eu.com \\ *** Université de Lyon, CNRS, INSA-Lyon, LaMCoS UMR5259, F-69621 Villeurbanne, France
}

\begin{abstract}
During the last decade, many new technical solutions dedicated to the comfort of automotive vehicle's drivers have raised, like Electrical Power Steering (EPS). To fulfill the more and more demanding requirements in terms of vibration and acoustics, the dynamic behavior of the whole steering is studied. The system is divided into dedicated finite elements (FE) describing the whole steering. The stress was first put on the gears models (worm gear and rack-and-pinion) and their anti-backlash systems as they have been identified as potential vibration sources. Mechanical non-linearities (clearances, non-linear stiffness) of the mechanical system are taken into account in these models. Then, this model allows simulating the transient response of the system to an input excitation. Each developed element is validated using a fitted experimental test bench. Then, the general model is correlated the same way. Hence models can be used to study the dynamic behavior of EPS systems or sub-systems.
\end{abstract}

Key words: Electrical Power Steering, Dynamic, Non Linear, Transient Response, Finite Elements, Gears, Worm Gear, Rack-and-Pinion

\section{Introduction}

Only a few studies are mentioned in the literature about the dynamic behavior of EPS. Most of the proposed mechanical models are based on the Bond Graph theory ${ }^{(1)-(4)}$, and take into account only one dimension of the system. Backlash, three-dimensional meshing stiffness of gears and couplings are neglected in these models. Other analytical models are proposed ${ }^{(5)}$. These models are based on the same basic assumptions. They mainly deal with the notions of road perception and stability of the system.

The work presented in this paper aims at studying and understanding the dynamic behavior of EPS in terms of generation of vibrations in the mechanical system (impacts due to backlash and other clearances) and of transmission of these EPS borne vibrations to the surroundings: the vehicle (through housings and tie rods) and the driver (through the column). Hence a three-dimensional modeling of the whole EPS taking into account the non linearities of this mechanism (gears backlash and clearances on other mechanical components like ball bearings for example) is developed in order to simulate the generation, the dissipation and the transmission of EPS borne vibrations.

EPS systems are first described so that the vibration context could be explained and objectives clearly set out. Then the approach is presented and the EPS modeling described. Assumptions, development, characterization and validation of dedicated FE are exposed. 
Finally an example of use of developed FE is proposed: the dynamic behavior of a simple mechanism is simulated to study the influence of ball bearings on it.

\section{Nomenclature}

$D$ : Balls diameter

$d_{m}$ : Bearing pitch diameter

$\{F\}:$ External load vector

$[M],[K],[C]:$ Mass, stiffness and damping matrices

$P_{d}$ : Bearing radial clearance

$\{q\},\{\dot{q}\},\{\ddot{q}\}$ Displacement, velocity and acceleration vectors

$r_{i} / r_{o}$ : Radii of inner/outer raceway curvature

$T:$ Torque

$t:$ Time

$Z:$ Number of balls

$z_{p}$ : Axial preload of the bearing (displacement)

$\delta:$ Relative configuration of non linear elements

\section{Subscripts}

ABLS: Anti-BackLash System

dof: degree of freedom

(C/P)-EPS: (Column/ Pinion)-Electric Power Steering

$F E$ : Finite Elements

\section{EPS systems}

EPS systems are described. The kinematics chain is detailed to introduce the modeling of each part of these systems.

\subsection{General description}

A steering is composed of several housings in which the kinematics chain components are enclosed. They link the steering wheel of the vehicle to the tie rods (Fig.1). The steering wheel turns the pinion gear; the pinion moves the rack in translation, from side to side. This motion applies steering torque to the kingpins of the steered wheels via tie rods and a short lever arm called the steering arm.

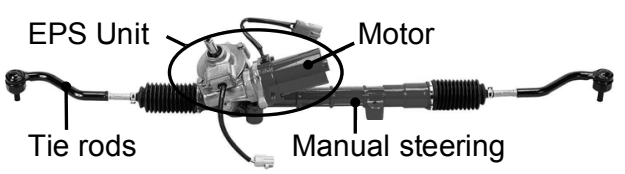

Fig.1 P-EPS system

Electric Power Steering systems were developed to help the driver to make wheels turn, vehicle becoming heavier. This technology allows better performances in terms of consumption than Hydraulic Power Steering, still in use on some vehicles. The assistance torque is delivered by an electrical motor controlled by a special unit. On most of these systems, this torque is applied through a worm gear (Fig.2) to the shaft, which can be the column (C-EPS) or the pinion (P-EPS).

The presented work is applied to P-EPS systems but can be used on other technologies. 
The kinematics chain of these systems is composed of three sub-systems: a manual steering, an EPS unit and a steering column.

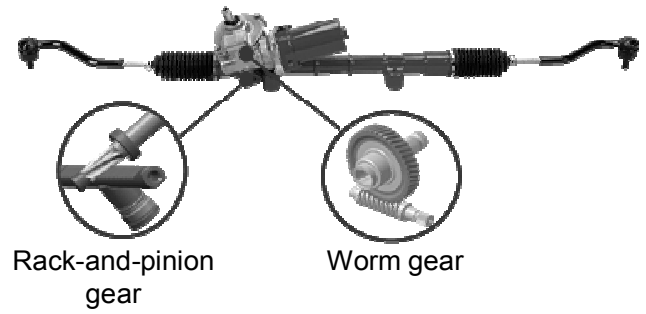

Fig.2 Gears of EPS systems

\subsection{The kinematics chain}

The manual steering sub-system of EPS is similar to a standard steering (Fig.3). It is composed of a rack-and-pinion gear, its anti-backlash system (the yoke line), a rack bush and tie rods.

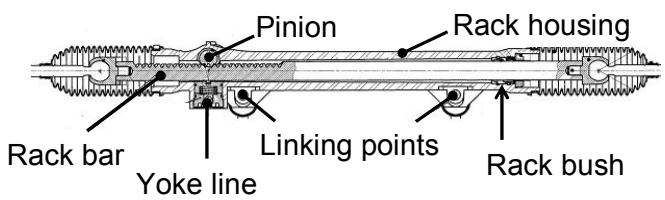

Fig.3 Manual steering

The yoke line (Fig.4) is composed of a support yoke assembly, a yoke plug (linked with the rack housing) and a spring.

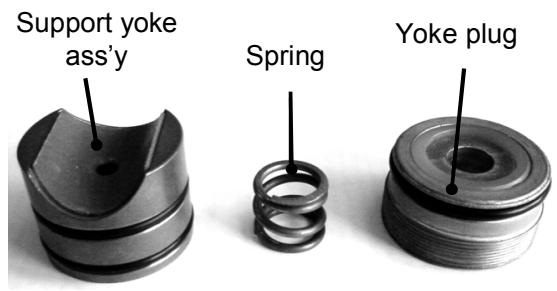

Fig.4 Yoke line components

In the EPS Unit, the assistance torque is given by an electrical motor and applied to the pinion via a worm gear (Fig.5). An anti-backlash system (ABLS) can be associated to this worm gear.

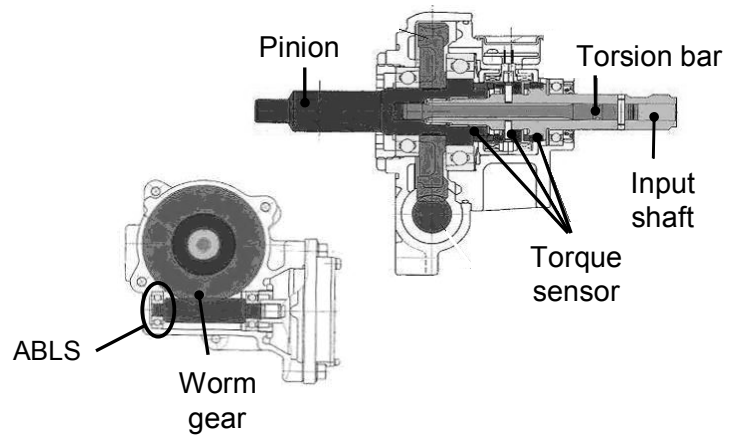

Fig.5 EPS Unit 
The kinematics chain of the EPS unit is also composed of three shafts: the pinion, the torsion bar and the input shaft (linked with the column). The assistance torque is controlled by a special unit according to the torsion of the bar and the velocity of the vehicle.

The column consists of two shafts, bearings and universal joints (Fig.6). It is linked to the vehicle through a dedicated housing.

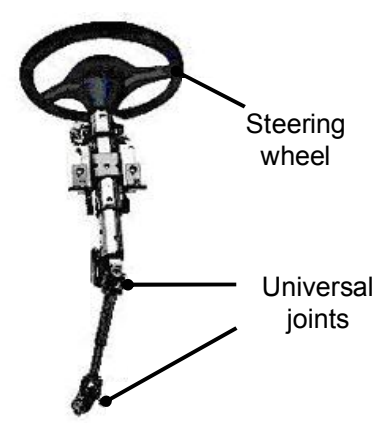

Fig.6 Column

\section{Vibrations in EPS}

The backlash of gears of EPS (Fig.2) is identified to be the main source of vibrations in these systems, coupled with clearances in their anti-backlash systems. Phenomenon like the transmission error will not be taken into account in this work. Let's consider the example of the sub-system composed of the pinion, the rack bar and the yoke line, in order to understand what is studied. The rack-and-pinion backlash is assumed to be nil (i.e. very small). The gear is then in configuration 3 (Fig.7). Increasing the torque in the sub-system, variation of the inter-axis due to possible retraction of the yoke line entails two contact configurations in the gearing: configuration 1 or 2 .
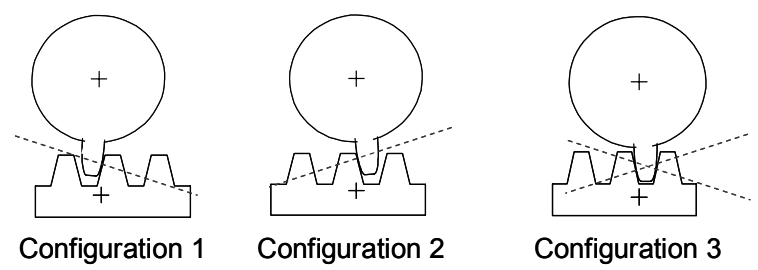

Fig.7 Meshing configurations

When the torque in the sub-system is zero again the gear comes back to configuration 3 . Hence impacts can occur at this time generating vibrations. These gear borne vibrations are transmitted to the housings, and then to the vehicle, through shafts and bearings. Hence the study of the EPS vibratory behavior implies low load configurations and a transient response of the system.

\section{Modeling of EPS}

\subsection{General description}

The whole EPS system is modeled in order to simulate its transient dynamical behavior being excited by external loads which can be, for example, an alternative movement on the steering wheel imposed by the driver, an excitation of tie rods from the road, or an assistance torque from the electrical motor (controlled by a dedicated electrical model) on 
the worm shaft. The system is divided into dedicated FE describing the whole steering. Displacements, velocities and accelerations at each node of the FE modeled structure are computed using the numerical method of Newmark ${ }^{(6)}$. The equation of motion to be solved is given in the form of matrices:

$$
[M]\{\ddot{q}\}+[C(\delta)]\{\dot{q}\}+[K(\delta)]\{q\}=\{F(t, \delta)\}
$$

Mechanical non-linearities (clearances, non-linear stiffness) of the system are taken into account in the developed FE models. Hence stiffness and damping matrices of the structure are not constant during the motion of the system: it depends on the configuration of each non linear FE. Hence impacts in the kinematics chain will be reproduced by the variation of the damping and stiffness matrices. The external load vector is variable in the same way and depends also on time (external loads).

\subsection{Developed FE models}

The stress was first put on the gears models and their anti-backlash systems as they have been identified as potential vibration sources. Then all necessarily FE to describe an EPS system were developed.

Hence developed FE models can be divided into:

- linear $\mathrm{FE}{ }^{(6)}$ : beams ${ }^{(8)}$, disks, mass, stiffness and damping elements, superelements;

- non linear FE: worm gear ${ }^{(6)(7)}$, rack-and-pinion gear ${ }^{(6)}$, ABLS spring, yoke line ${ }^{(9)}$, universal joints ${ }^{(6)}$ and ball bearings ${ }^{(10)-(13)}$.

The developed models are based on a geometrical description of the considered part of the EPS. The assumption of small displacements is retained. Only small vibratory displacements about a working point are considered. Hence each non linear stiffness of an element is computed as a tangent stiffness matrix linearized about the working point. This tangent stiffness is updated at each time step of the transient response computing. To avoid high numerical discontinuities when the non linear stiffness is modeled as a piecewise linear stiffness, it is approximated by a continuous function ${ }^{(6)}$. For example the meshing stiffness of gears taking into account backlash is approximated by such a function ${ }^{(7)}$.

For each non linear FE taking into account clearances, load on EPS is considered to be low enough so that all parts in contact are considered as rigid bodies except at the contacts. Their geometry is assumed to be perfect.

\subsection{Determination of models parameters}

Each FE used to build a complete EPS model is configured by different parameters which can be:

- geometrical parameters (clearances, angles, lengths, ...);

- stiffness and damping parameters;

- mass and inertia parameters.

These parameters are obtained from:

- technical drawings (geometry, mass and inertia);

- experimental characterization (stiffness, clearances);

- specific static meshing tools (meshing stiffness, application point and line of action for rack-and-pinion and worm gears $\mathrm{FE}^{(14)}$, mean values). 
Damping parameters are tuned using correlation studies between simulation and experimental dynamical tests.

\subsection{Validation of the models}

Each developed FE is validated with experimental and/or analytical results. Validation data can be obtained from quasi-static relations and dynamical tests. The element can be isolated from its surroundings to be validated. It can also be connected with other validated elements in order to model a part of an EPS. In this case the dynamical behavior of the modeled structure validates the new FE.

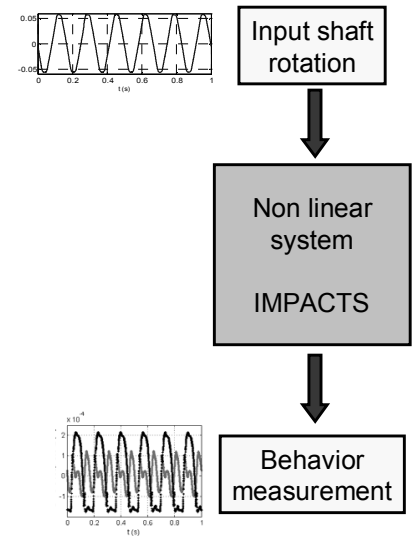

Fig.8 Experimental dynamical tests principle

At the end the assembly of all the FE to build an EPS model is validated by correlation with dynamical experimental tests. These tests (Fig.8) consist in applying an external load on the system (rotation on the steering wheel or on the input shaft) at a low frequency (under $10 \mathrm{~Hz}$ ). Non linearities of the system generate impacts in the structure which make the system to be excited at high frequencies. The dynamical behavior of the system is measured and compared with simulation results of the modeled test bench. For example the behavior of the worm shaft, lead by the worm gear, the ABLS spring and ball bearings is observed (Fig.9).
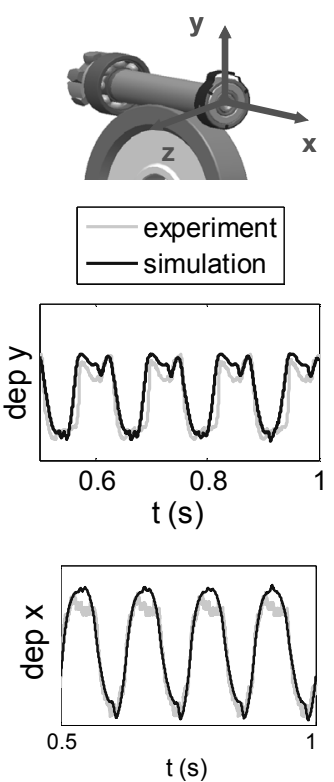

Fig.9 Worm displacement along $\mathrm{Y}$ and $\mathrm{X}$ axes 


\section{Ball bearings modeling: influence on the behavior of a simple mechanism}

The dynamic behavior of a simple mechanism is simulated in order to study the influence of ball bearings modeling as non linear boundary conditions for the kinematics chain (non linear stiffness, clearance).

\subsection{Studied mechanism}

The studied mechanism (Fig.10) is a worm gear with backlash. Node 1 is the center of the wheel. Node 2 is the center of the worm shaft which rotates in two ball bearings placed at each of its ends. Nodes 3 and 4 are the nodes associated with the inner ring and the outer ring of the considered ball bearing. It is the same for nodes 5 and 6 .

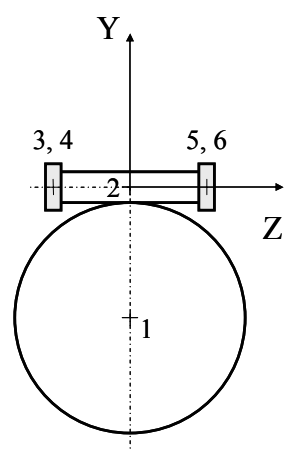

Fig.10 Studied mechanism
Table1 Bearings 1 and 2 characteritics

\begin{tabular}{|c|c|}
\hline Reference & Test bearing \\
\hline $\mathrm{D}(\mathrm{mm})$ & 4.7625 \\
\hline $\mathrm{d}_{\mathrm{m}}(\mathrm{mm})$ & 17.6505 \\
\hline $\mathrm{Z}$ & 7 \\
\hline $\mathrm{r}_{\mathrm{i}}(\mathrm{mm})$ & 2.465 \\
\hline $\mathrm{r}_{\mathrm{o}}(\mathrm{mm})$ & 2.536 \\
\hline $\mathrm{P}_{\mathrm{d}}(\mu \mathrm{m})$ & 11 \\
\hline Mass $(\mathrm{kg})$ & 0.0190 \\
\hline
\end{tabular}

The wheel is built with a disk FE and rotates about its axis; it is its only free dof. The worm shaft is built with two beam FE (two nodes with all dof free). An external load is applied on the worm at node 5; it is an alternative torque of amplitude $3 \mathrm{Nm}$ and frequency $5 \mathrm{~Hz}$ :

$$
T=3 \sin (2 \pi .5 t)
$$

A stiffness $(1000 \mathrm{Nm} / \mathrm{rad})$ is applied on rotation on the wheel as boundary condition of the mechanism. Bearings are clamped at their outer rings (rigid housing). Three configurations of the mechanism are tested:

In configuration 1 ball bearings are considered as linear boundary conditions. Translational stiffness of bearings along axes $\mathrm{X}, \mathrm{Y}$ and $\mathrm{Z}$ are:

$$
\left[K_{1}\right]=\left[K_{2}\right]=1.10^{7} \times\left[\begin{array}{ccc}
8.3 & 0 & 0 \\
0 & 8.3 & 0 \\
0 & 0 & 2.3
\end{array}\right]_{(X, Y, Z)}
$$

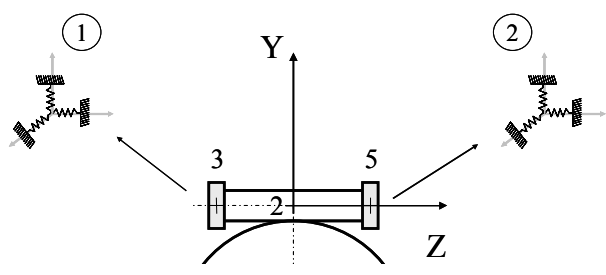

Fig.11 Studied mechanism - configuration 1

In configuration 2 ball bearings are modeled using the dedicated FE: non linear stiffness and radial clearance are taken into account. Bearings preload is (in meters): 


$$
\begin{aligned}
& z_{p_{1}}=-8.10^{-5} \mathrm{~m} \\
& z_{p_{2}}=8.10^{-5} \mathrm{~m}
\end{aligned}
$$

Hence the initial elementary stiffness of bearings is, along and around axis $\mathrm{X}, \mathrm{Y}$ and $\mathrm{Z}$ :

$$
\begin{gathered}
{\left[\begin{array}{cccccc}
8,3 & 0 & 0 & 0 & 0,025 & 0 \\
0 & 8,3 & 0 & -0,025 & 0 & 0 \\
0 & 0 & 2,3 & 0 & 0 & 0 \\
0 & -0,025 & 0 & 9.10^{-5} & 0 & 0 \\
0,025 & 0 & 0 & 0 & 9.10^{-5} & 0 \\
0 & 0 & 0 & 0 & 0 & 0
\end{array}\right]_{(X, Y, Z)}} \\
{\left[\begin{array}{ccccccc}
8,3 & 0 & 0 & 0 & -0,025 & 0 \\
0 & 8,3 & 0 & 0,025 & 0 & 0 \\
0 & 0 & 2,3 & 0 & 0 & 0 \\
0 & 0,025 & 0 & 9.10^{-5} & 0 & 0 \\
-0,025 & 0 & 0 & 0 & 9.10^{-5} & 0 \\
0 & 0 & 0 & 0 & 0 & 0
\end{array}\right]_{(X, Y, Z)}} \\
\\
\end{gathered}
$$

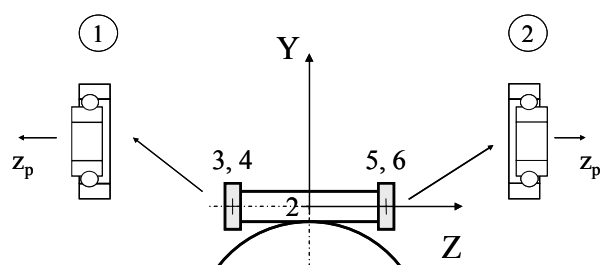

Fig.12 Studied mechanism - configuration 2

In configuration 3 ball bearings are modeled using the dedicated FE as in configuration 2. Bearings are not preloaded. Hence initial elementary stiffness of bearings is nil.

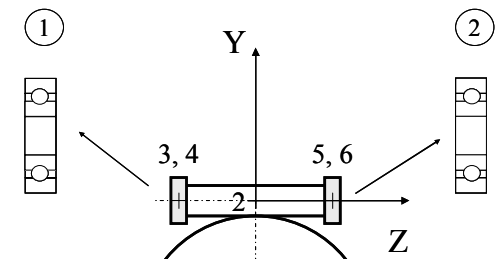

Fig.13 Studied mechanism - configuration 3

The time response of the mechanism in each three configurations is computed from Eq.(1). Displacements, velocities and accelerations of each free dof are computed. Stiffness matrices of ball bearings are also stored during the computation.

The worm gear FE model is given by Bordegaray ${ }^{(6)(7)}$ and formulated in appendix. The ball bearing FE model is also detailed in appendix. 


\subsection{Stiffness and displacements study}

As an example of results the evolution of the stiffness of bearings 1 and 2 along axis $Z$ (diagonal terms of the stiffness matrices) is observed for each defined configuration (Fig.14). Bearings stiffness is constant in configuration 1. If bearings are modeled using dedicated FE (configurations 2 and 3) stiffness changes with time according to the displacements of the inner ring of the bearing.

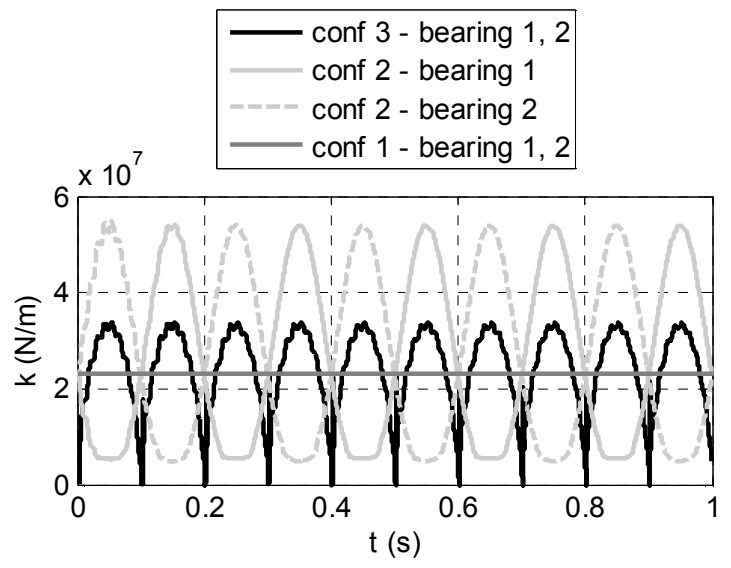

Fig.14 Bearings stiffness along axis Z evolution

In configuration 2 the applied preload on bearings allows keeping a high stiffness along axis $\mathrm{Z}$ in the mechanism (preload of opposite sign on each bearing) as in configuration 1 . That is not the case in configuration 3: bearings are not preloaded. The mechanism stiffness tends towards low values in this configuration along axis $\mathrm{Z}$ due to the clearance of the bearings.

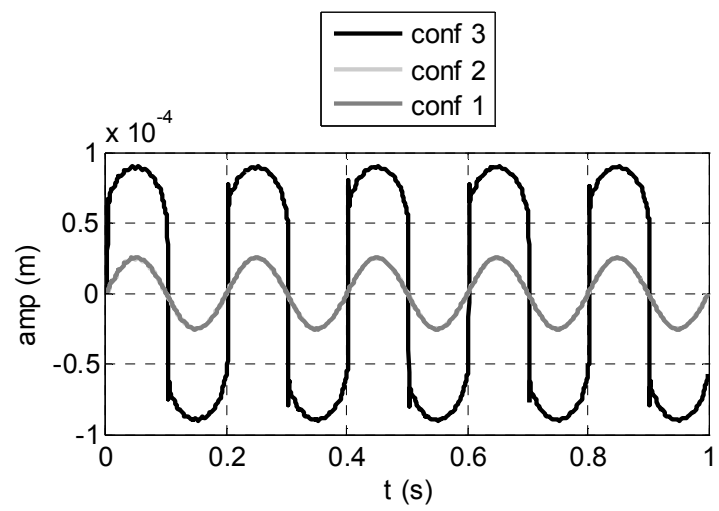

Fig. 15 Worm displacement along axis $\mathrm{Z}$ at node 2

This phenomenon can be observed on the displacements of the worm shaft along axis $\mathrm{Z}$ (Fig.15). Displacements simulated in configurations 1 and 2 are similar. Hence stiffness along axis $\mathrm{Z}$ in these both configurations keeps high. On the other hand this displacement is much more important in configuration 3 . Hence the mechanism sometimes works with a low stiffness along $\mathrm{Z}$.

\subsection{Frequency analysis}

The torque imposed on the mechanism leads to the rotation of the wheel. The frequency analysis of the acceleration of this movement between 0 and $150 \mathrm{~Hz}$ as example (Fig.16) shows a first frequency at $5 \mathrm{~Hz}$ corresponding to the frequency of the excitation of the 
mechanism, and also a second higher frequency at about $90 \mathrm{~Hz}$ for the three configurations.

This frequency corresponds to a fundamental mode of the structure making the wheel and the worm rotate about their axes and strongly linked with the torsion stiffness connected on the wheel, but not only. Indeed the contact load in the worm gear is three dimensional. Hence stiffness of the whole structure influences this movement and so the associated mode, for example the meshing stiffness in the worm gear or the bearings stiffness. This explains why the identified frequency is not the same in the three configurations.

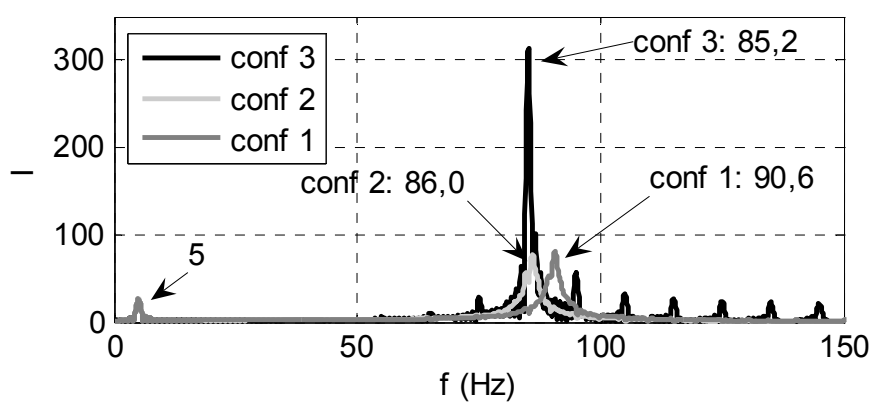

Fig.16 FFT of the wheel rotation (acceleration)

Dedicated ball bearings FE (configurations 2 and 3) modifies the behavior of the mechanism compared with configuration 1 . The identified frequency in configuration 1 is higher. One can also note that this frequency is not exactly the same in configurations 2 and 3 , which can be explain considering the meanly lower stiffness of a not preloaded bearing compared with a preloaded one, as showed previously.

One can note that configuration 3 FFT contains harmonics linked with the main movement frequency at $5 \mathrm{~Hz}$. Hence the mechanism is highly non linear in this configuration due to clearances: bearings are not preloaded.

It is also interesting to compare the previously identified frequencies (Fig.16) with computed natural frequencies of the structure. The structure is non linear: its natural frequencies are computed for a direct contact in the worm gear and for the initial value of bearings stiffness in configuration 2. It is not computed in configuration 3 because of the nil initial value of bearings stiffness.

In configuration 1, the computed frequency is $90.5 \mathrm{~Hz}$, which corresponds to the value obtained by FFT of the simulated acceleration of the wheel rotation. In configuration 2, it is $78 \mathrm{~Hz}$, which is lower than the value obtained by FFT $(86 \mathrm{~Hz})$. This means the bearings stiffness changes during the time response computing of the structure and does not keep equal to the initial value used for the frequency response computing. Hence the computed natural frequency $78 \mathrm{~Hz}$ is not representative of the stiffness of the mechanism under working conditions (applied external load). The time response analysis should be preferred to analyze the dynamic behavior of this non linear mechanism.

\section{Summary}

The developed models allow simulating the dynamic behavior of EPS systems. Dedicated FE were correlated separately and/or in small assemblies. Once correlated, they were assembled to model complete EPS. The recent correlations on the whole system show good results in terms of dynamic movements at different positions and also in terms of shocks apparitions due to non linearities (mainly in gears). The models have been developed to be easily adapted to any kind of system. 
The dynamic behavior of a simple mechanism modeled with dedicated FE is simulated and studied. This study, for the three defined configurations, shows that bearings stiffness is very influent on the mechanism behavior. It shows the interest to take into account the bearings radial clearance, its preload and non linear stiffness due to geometry, all the more since some bearings are not preloaded in EPS systems.

New developments aim at enlarge the simulation to the fixation points. Tie-rods and columns will thus be modeled. The link between the many results and their impact on the noise perception will also be studied with the development of new analysis tools.

\section{Acknowledgement}

The authors wish to thank JTEKT Europe for its technical and financial support.

\section{References}

(1) Taheri S.M., Karim S., Mohamed M.G., Dynamic analysis of a hydraulically assisted rack and pinion power steering system using bond graph, Proc. of the ASME Conference on ESDA, London, England, 1994.

(2) Zhang Q., Wu D., Reid J.F., Benson, E.R., Model recognition and validation for an off-road vehicle electrohydraulic steering controller, Mechatronics Vol.12 (2002) pp.845-858.

(3) Simon P., Numerical Simulation of Electric Power Steering (EPS) System, Koyo Engineering Journal English Edition Vol.161E (2002) pp.52-56.

(4) Badawy, A., Zuraski J., Bolourchi F., Chandy A., Modeling and analysis of an electric power steering system, SAE Technical Paper Series 1999-01-0399 (1999).

(5) Proca, A.B., Keyhani A., Identification of power steering system dynamic models, Mechatronics Vol.8 (1998) pp.255-270.

(6) Bordegaray C. Dynamic behavior of EPS systems: theory and experiment (in French), PhD, ISAL007, 2007.

(7) Bordegaray C., Ferraris G., Guingand M., de Vaujany J.P., Dynamic behavior of a worm gear, Proc. of ASME Design Engineering Technical Conferences and Computers and Information in Engineering Conference, Long Beach, California USA, Ref : DETC2005-85198. 2005.

(8) Couderc P., Dynamic behavior of automotive transmissions (in French), PhD, ISAL0063, 1997.

(9) Besson F., Ferraris G., Guingand M., de Vaujany J.P., Dynamical model of the yoke line of EPS (in French), Proc. of 16th VCB conference, Ecully, FRANCE, 2008.

(10) Lim T.C., Singh R., Vibration transmission through rolling element bearings, part I: Bearing stiffness formulation, Journal of Sound and Vibration Vol.139 $N^{\circ} 2$ (1990) pp.179-199.

(11) Lim T.C., Singh R., Vibration transmission through rolling element bearings, part II: system studies, Journal of Sound and Vibration Vol.139 N² (1990) pp.201-225.

(12) Houpert L., A uniform analytical approach for ball and roller bearings calculations. Journal of Tribology Vol.119 (1997) pp.851-858.

(13) Lahmar F., Gear meshing and bearings dynamical interaction (in French), PhD, ISAL0020, 2000.

(14) Hiltcher Y., Guingand M., de Vaujany J.P., Load sharing of worm gear with a plastic wheel, Journal of Mechanical Design Vol.129 $N^{\circ} 1$ (2007) pp.23-30. 


\section{Appendix 1: ball bearings modeling}

The ball bearing FE model is based on the model given by Lim and Singh ${ }^{(10)-(12)}$ and detailed by Lahmar ${ }^{(13)}$. It was adapted to considered as reference position for the bearing the position where inner ring and outer ring are centered on the same point. Hence the reference model ${ }^{(10)}$ is modified only in terms of the expression of the $j$ th rolling element displacements (Fig.17):

$$
\begin{gathered}
\delta_{z j}=\left(\delta_{z}+z_{p}\right)+R_{j}\left(\beta_{x} \sin \psi_{j}-\beta_{y} \cos \psi_{j}\right)-A_{0} \sin \alpha_{0} \\
\delta_{r j}=\delta_{x} \cos \psi_{j}+\delta_{y} \sin \psi_{j}
\end{gathered}
$$

With (Fig.17):

$$
\cos \alpha_{0}=1-\frac{P_{d}}{2 A_{0}}
$$
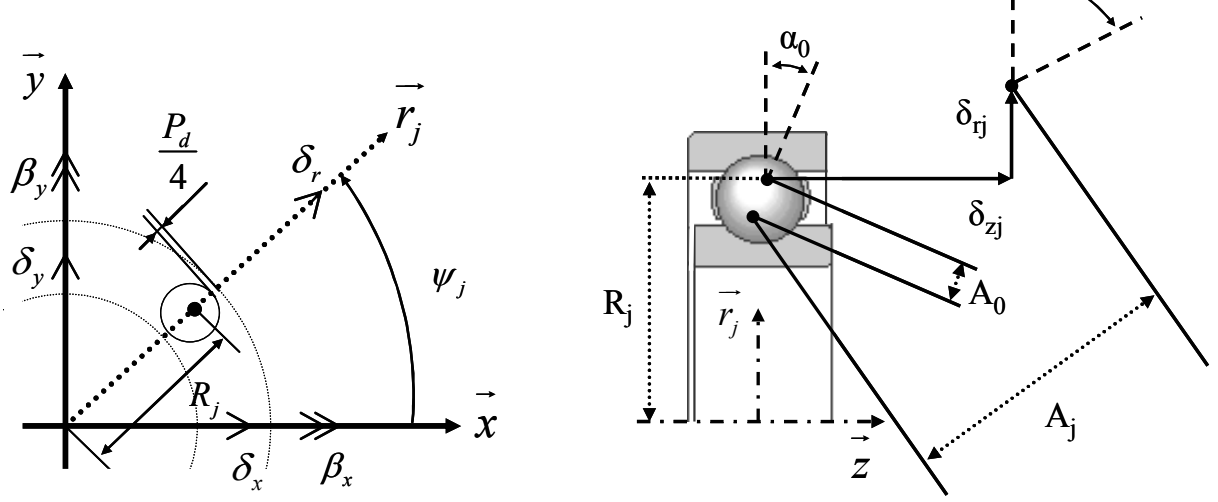

Fig.17 Ball bearings details

\section{Appendix 2: worm gear meshing modeling}

The worm gear FE model is given by Bordegaray ${ }^{(6)(7)}$. Both wheel and worm are considered as rigid bodies except along a direction normal to the contact surfaces: therefore the gears are interconnected by a spring-damper system. The displacements of any gear point can be written according to the displacements and rotations of the worm and wheel centers. Both worm and wheel are allowed to translate in three dimensions and to rotate along three axes: the model has two nodes and six degrees of freedom per node. Three meshing configurations have to be considered because of backlash (Fig.7). Each configuration $i$ leads to the expression of the stiffness meshing:

$$
K_{e n g_{i}}=k_{i} \cdot \vec{\sigma}_{i}^{t} \cdot \vec{\sigma}_{i}
$$

Where:

- $k$ is the mean value of the meshing stiffness obtained from the specific static meshing tool ${ }^{(14)}$. This value depends on the meshing configuration, and so on the backlash of the worm gear.

- $\vec{\sigma}$ is a vector composed of geometrical parameters of the gear mechanical component (inter axis, helix angles, pressure angle, ...). This vector depends on the meshing configuration, and so on the backlash of the worm gear. 